\title{
Influence of packing factor on coercivity of particle arrays
}

\author{
Ching-Ray Chang ${ }^{\mathrm{a}, *}$, Jyh-Shinn Yang ${ }^{\mathrm{b}}$ \\ a Department of Physics, National Taiwan University, Taipei, Taiwan, ROC \\ ${ }^{b}$ Dirision of General Education, National Taiwan Ocean Unicersity, Keelung, Taiwan, ROC
}

\begin{abstract}
The influence of the packing factor on the coercivity of particle arrays has been calculated. The interaction fields are treated as the results of an infinite array of rectangular prisms. Temperature and sweep rate have also been considered, and it was found that the nonlinear dependence of the coercivity at small packing can result from the interaction field.
\end{abstract}

\section{Introduction}

The relationship between packing factor and coercivity for an assembly of fine particles is an old but difficult problem since complicated, many-body effects are involved. The famous formula $H_{c}(p)=(1-p) H_{c}(0)$ does not always agree with experimental results, in particular, at small packing factor [1]. Although the similar expression $H_{c}(p)=A(1-p)+B$ often works better, where $A$ is attributed as the coercivity arising from the shape and $B$ is the contribution of the crystalline anisotropy and the shape of the sample [2]. However, it did not explain the nonlinear dependence of the coercivity at small packing density. The interaction fields among the particles are long range force, but they are usually calculated either by a mean field method [2] or numerical methods [1]. However, the mean field model fails in very dilute situations and the numerical method, as a rule, requires a lot of computation power. Moreover, the finite size effect of the particles is usually neglected, i.e., the interaction fields are treated as a dipolar field for simplicity. Thus, the attempts to understand the coercivity dependence of the packing factor become unsatisfactory for appropriately describing the nature of the interaction. Here we use the Fourier method to calculate the interaction field of a three-dimensional infinite array of rectangular prisms. The interaction fields dependence on the packing density is found to be quite nonlinear, in particular, for pancake-like particles. Therefore, the nonlinearity of the dependence of the coercivity at small packing density can result from the interaction field alone. For pancake-like particles, the interaction fields even become positive at very small packing densities. Because of the finite size of the particles, interaction among needle-like

\footnotetext{
Corresponding author. Email: crchang@phys.ntu.edu.tw; fax: $+886-2-363-9984$
}

and pancake-like particles shows quite different dependence of the packing factor. With the consideration of the thermal activation, we can also easily analyze the influence of temperature on the coercivity. Sweep rate has also been investigated, but the nonlinear dependence of the coercivity at the small packing factor is insensitive to the thermal effects.

\section{Model}

We assumed single-domain particles are situated at a three-dimensional tetragonal lattice. There are particles in each lattice site separated at distance $g$. The central particle was considered as a spheroidal particle, but all the surrounding particles are treated approximately as prisms with the same aspect ratio for convenience. The square cross section of the prism is $w^{2}$, and its height $t$. The magnetization of each prismatic particle is initially saturated along the direction of the applied field $\boldsymbol{H}_{\mathrm{a}}$. The crystalline anisotropy energy of the particles is assumed to be along the direction of the applied field, and the shape anisotropy energy assumed to be small enough to avoid to form an easy cone for the oblate spheroidal particles [3].

The interaction field is the summation of the interaction field of all the other prisms acting on the central prism [4]. Invoking the superposition principle, the total interaction field for the central prism can be treated as the sum of fields from many infinite two-dimensional arrays. The field of each layer can be calculated from the Fourier series method. For an infinite two-dimensional array of the prisms separated at distance $g$, the period of the magnetization is $w+g$, and it is an even function along either $x$ or $y$ directions. Thus, it can be expanded as a two-dimensional Fourier series,

$$
M(x, y)=\sum_{n=0}^{\infty} \sum_{m=0}^{\infty} M_{n m} \cos k_{n} x \cos k_{m} y,
$$


where $k_{l}=(2 / \pi) /(w+g), l=n, m$. Solving the Poisson equation $\nabla^{2} \phi=4 \pi \nabla \cdot \boldsymbol{M}$, and $\boldsymbol{H}_{t}=-\nabla \phi$, it follows

$$
\begin{aligned}
\frac{H_{r .:}}{4 \pi M_{s}}= & -\frac{w^{2}}{(w+g)^{2}} \\
& -\frac{4 w}{(w+g) \pi} \sum_{n=1}^{x} \frac{1}{n} \sin \frac{k_{n} w}{2} F\left(k_{n}, t, g\right) \\
& -\frac{4}{\pi^{2}} \sum_{m, n=1}^{x} \frac{1}{m n} \sin \frac{k_{n} w}{2} \sin \frac{k_{m} w}{2} F\left(k_{n m}, t, g\right),
\end{aligned}
$$

where

$F(k, t, g)=\exp \left(-\frac{k t}{2}\right) \frac{1-\exp (-k g)}{1-\exp [-k(t+g)]}$,

and $k_{n m}=\left\{k_{n}^{2}+k_{m}^{2}\right\}^{1 / 2}$. Because we are interested in the local interaction field from the other prisms, the magnetostatic field at the center prism

$$
\frac{H_{\mathrm{p} .:}}{4 \pi M}=-\frac{2}{\pi} \tan ^{-1}\left[\frac{w^{2}}{t \sqrt{2 w^{2}+t^{2}}}\right]
$$

should be deducted. Therefore, the total field $\boldsymbol{H}$ acting on the central particle will be $\boldsymbol{H}_{\mathrm{a}}+\boldsymbol{H}_{t}-\boldsymbol{H}_{\mathrm{p}}$.

To include the thermal effects, we treat the particles as having two levels [5] which are occupied with probabilities $n_{1}$ and $n_{2}=1-n_{1}$ respectively. Its evolution is given by the master equation

$\dot{n}_{1}=-\kappa_{12} n_{1}+\kappa_{21} n_{2}=-\dot{n}_{2}$

where $\dot{n}_{i}=\mathrm{d} n_{\mathrm{i}} / \mathrm{d} t$ and $\kappa_{i j}=f_{0} \exp \left(-Q_{i} / k_{\mathrm{B}} T\right)$ is the rate of thermally activated transitions from the $i$ th to the $j$ th level. For the prefactor we choose the value $f_{0}=10^{10} \mathrm{~Hz}$, $k_{\mathrm{B}}$ is the Boltzmann constant, $T$ is temperature and $Q_{i}$ is the barrier height to be overcome on departure from the $i$ th well. We shall assume that the applied field driving the hysteresis loop is in the z-direction, $\boldsymbol{H}_{\mathrm{a}}=\left[0,0, H_{\mathrm{a}}(t)\right]$, $H_{i}(t)=H_{0} \cos 2 \pi f t$, where $f$ is the sweep rate and the amplitude $H_{0}$ is greater than the nucleation field of the system. For perfectly aligned particles, the barrier heights are given by the well known formula $Q_{i}=K V(1 \pm$ $H\left(H_{\mathrm{k}}\right)^{2}$, where $H_{\mathrm{k}}$ is the anisotropy field of the particle.

\section{Results and discussions}

Packing density decreases as the separation distance $g$ increases. Therefore, we can easily study the influences of packing density from the variation of $g$ in our calculations. The interaction field of needle-like particles is almost linearly varying with the packing factor, and thus coercivity dependence on the packing factor is also linear. However, for pancake-like particles, the interaction field dependence on the packing factor becomes quite nonlinear, its coercivity dependence deviates from the linear predic-

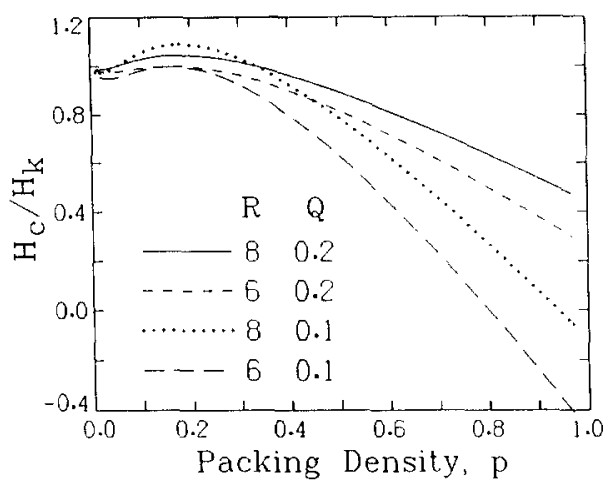

Fig. 1. Reduced coercivity $\left(H_{\mathrm{c}} / H_{\mathrm{k}}\right)$ dependence of packing density for pancake-like particles, where $p=\left[w^{2} t\right] /\left[(w+g)^{2}(t\right.$ $+g)$ ]. The aspect ratio $R=w / t$ and the quality factor $Q=$ $K_{i} \cdot /\left(2 \pi M_{?}^{2}\right)$.

tion substantially (Fig. 1). In particular, for very dilute packing density, e.g. $p \leq 0.05$, a bump in the coercivity dependence resulted from the small positive interaction field at this situation. The effects of the temperature and sweep rate only shift the whole graphs but they cannot change the dependence of packing density significantly. In general, the higher temperature and the slower sweep rate usually give smaller coercivity. We have also calculated the influence of the shape on the reduced coercivity ( $H_{c} / H_{\mathrm{k}}$ ) for the same packing density (Fig. 2). Needle-like particles are more sensitive to the packing density than pancake-like particles. It was shown that the smallest reduced coercivity appears at the range of aspect ratio $1-3$.

It has been shown that the nonlinear dependence of coercivity on the packing density for pancake-like particles comes from the interaction field only. However, for needle-like particles, it should be explained otherwise [6], e.g. by the agglomeration of the particles or irreversible breaking of the particle during the dilution process. The possibility of the formation of an easy cone was neglected for pancake-like particles, but it deserves further investigation.

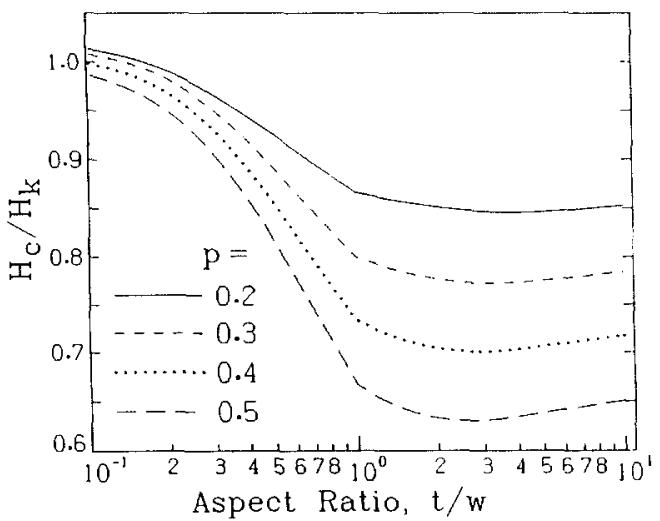

Fig. 2. The relationship between the reduced coercivity and the aspect ratio for different packing densities $p$. Here $Q=1$. 


\section{Acknowledgements}

This research was support in part by the National Science Council of the Republic of China under grant No. NSC 84-2112-M002-17.

\section{References}

[1] J.E. Knowles, IEEE Trans. Magn. 21 (1985) 2576.

[2] C.-R. Chang and J.-P. Shyu, J. Magn. Magn. Mater. 120 (1993) 197.
[3] If shape anisotropy energy is of the same order as crystalline anisotropy energy, there is a possibility of forming an easy cone at oblate spheroidal particles, which was not considered at present.

[4] C.-R. Chang and J.-S. Yang. CompuMag 95, Berlin, Germany (1995), PA1-7.

[5] J.J. Lu, H.L. Huang, C.-R. Chang and I. Klik, J. Appl. Phys. 74 (1994) 5499.

[6] C.-R. Chang and J.-P. Shyu, J. Appl. Phys. 73 (1993) 6659. 